(Physiologisches Laboratorium in Bonn.)

\title{
Prüfung der Harnstoffanalyse Hüfner's mit Hülfe der von uns verbesserten Methode Bunsen's.
}

\author{
Von
}

\section{E. Pflüger und K. Bohland.}

Nachdem durch Pflüger und Schenck $\mathbf{k}^{\mathbf{1}}$ ) der Beweis erbracht wurde, dass $H$ üfner's Methode einen zn kleinen Werth für den Gesammtstickstoff des menschlichen Harnes liefert, blieb die Frage zu entscheiden, ob wenigstens der Harnstoff nach ibr richtig bestimmt werden könne, wie das in der aus Hüfner's Laboratorium neuerdings hervorgegangenen Arbeit von Jacoby ${ }^{2}$ ) behauptet wird.

Da wir die Harnstoffanalyse nach Bunsen wesentlich in neuester Zeit verbessert haben, so lässt sich jene Frage jetzt mit Aussicht auf Erfolg in Angriff nehmen.

Um dem Leser die Einsicht in unsere Untersuchung zu erleichtern, fassen wir dieselbe übersichtlich in drei Tabellen zusammen.

Tabelle I soll die nach Hüfner's Vorschrift mit unverditnnter Knop'scher Lauge ausgeführten Analysen geben, von denen ein Theil dureh Herrn F. Schenck im hiesigen Laboratorium wie wir wissen in correcter Weise bearbeitet worden ist.

Tabelle II enthält die mit denselben Harnen ausgeführten Kohlensäureanalysen zur Ermittlung des Harnstoffs nach der verbesserten Bunsen'schen Methode.

Tabelle III fasst die wesentlichen Resultate aus Tabelle II und III vergleichend zusammen und berïcksichtigt zugleich den Gesammtstickstoff.

In einem Nachtrag "analytischer Belege" wollen wir die Daten bringen, die sich zur Aufnahme in die Tabelle nicht eignen und deren Kenntniss doch von manchem Leser gewünscht wird,

1) Dies Archiv. XXXVIII. p. 325.

2) Fresenius, Zeitschr. f. analyt. Chemie. 21,606.

E. Pflüger, Archiv f. Phygiologie. Ba. XxxIX. 


\begin{tabular}{|c|c|c|c|c|c|}
\hline $\begin{array}{l}\infty \\
\infty \\
\stackrel{\infty}{*} \\
\tilde{\sigma}\end{array}$ & 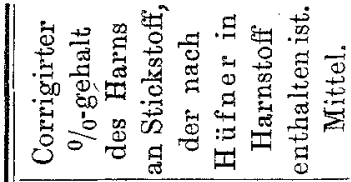 & $\overbrace{}^{\infty}$ & $\underbrace{8_{0}^{-1}}_{-1}$ & $\underbrace{\underbrace{\infty}_{-1}}$ & $\begin{array}{r}\stackrel{g}{8} \\
0 \\
\end{array}$ \\
\hline $\begin{array}{l}1 \\
1 \\
0 \\
0 \\
0 \\
0 \\
0 \\
0 \\
01 \\
0\end{array}$ & 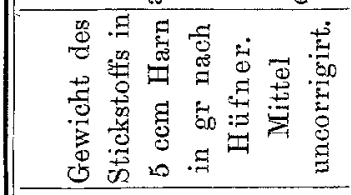 & 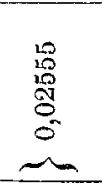 & 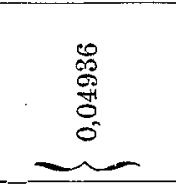 & $\begin{array}{r}0 \\
19 \\
5 \\
0 \\
0 \\
0 \\
\end{array}$ & 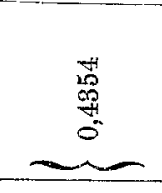 \\
\hline 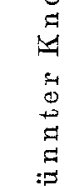 & 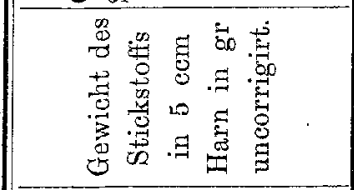 & $\begin{array}{ll}20 & 10 \\
\text { iv } & 00 \\
2 & 10 \\
01 & 9 \\
0 & 0 \\
0 & 0\end{array}$ & $\begin{array}{llll}\infty & 0 & 0 \\
4 & 8 & 1 & 8 \\
0 & 8 & 0 \\
\sharp & 0 & 0 \\
0 & 0 & 0 \\
0 & 0 & 0 & 0\end{array}$ & 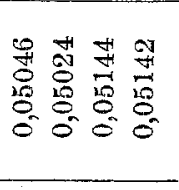 & 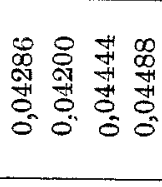 \\
\hline 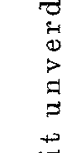 & 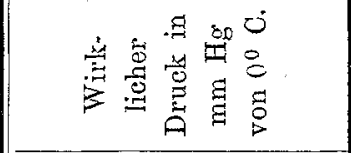 & 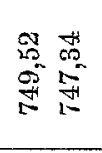 & 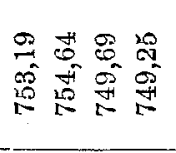 & 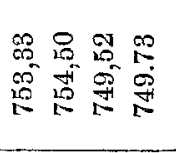 & 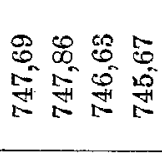 \\
\hline 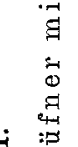 & 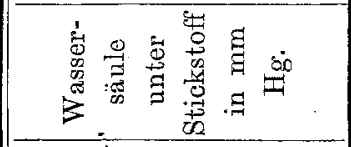 & 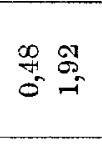 & 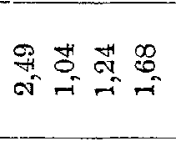 & $\begin{array}{l}200 \\
60 \\
6\end{array}$ & 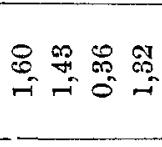 \\
\hline 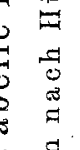 & 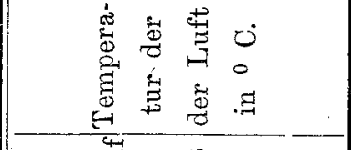 & $\infty$ & $\begin{array}{cccc}0 & 0 & 0 \\
\infty & \infty & \infty & \infty \\
0 & \infty\end{array}$ & $\begin{array}{cccc}0 & 0 & 0 & 0 \\
\infty & \infty & \infty & \infty \\
\infty & \infty\end{array}$ & 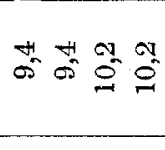 \\
\hline ב & 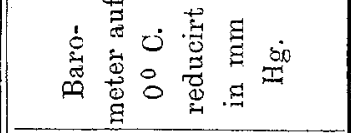 & 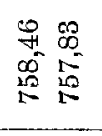 & 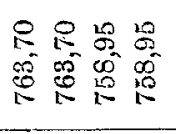 & 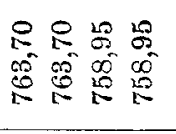 & 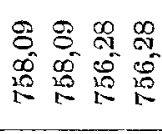 \\
\hline $\begin{array}{l}-9 \\
0 \\
0 \\
0 \\
0 \\
0 \\
0\end{array}$ & 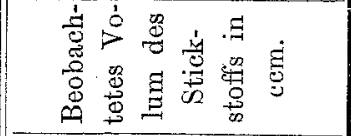 & $\underbrace{+}_{-1} \stackrel{4}{\infty}$ & 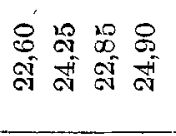 & 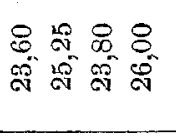 & 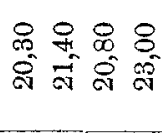 \\
\hline $\begin{array}{l}E \\
\stackrel{E}{g} \\
g \\
0 \\
0\end{array}$ & 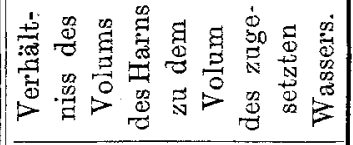 & $\stackrel{\infty}{\ddot{*}} \ddot{\ddot{*}}$ & 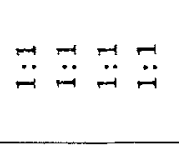 & $\ddot{\ddot{n}} \ddot{\ddot{r}} \ddot{\ddot{r}}$ & $\ddot{\ddot{n}} \ddot{\ddot{n}} \ddot{\ddot{n}}$ \\
\hline 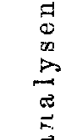 & 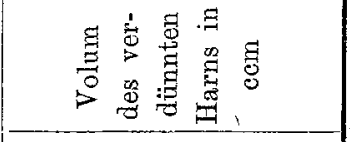 & 1008 & 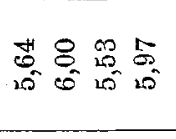 & 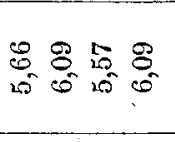 & $\begin{array}{llll}0 & 0 & 0 & 8 \\
0 & 0 & 8 \\
15 & 0 & 0 & 0\end{array}$ \\
\hline $\begin{array}{c}+\infty \\
+ \\
0 \\
+\end{array}$ & surte [H səp rəurmen & $\exists=$ & $Z==$ & $\exists==$ & $\Xi \approx \therefore \quad \approx$ \\
\hline 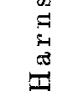 & 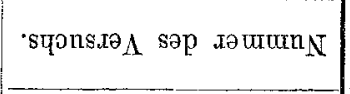 & $\sqrt{-10}$ & $T_{+1200}$ & $\underbrace{\infty}_{\infty}$ & $=\underset{ \pm}{ \pm}$ \\
\hline & • & & & & - \\
\hline
\end{tabular}




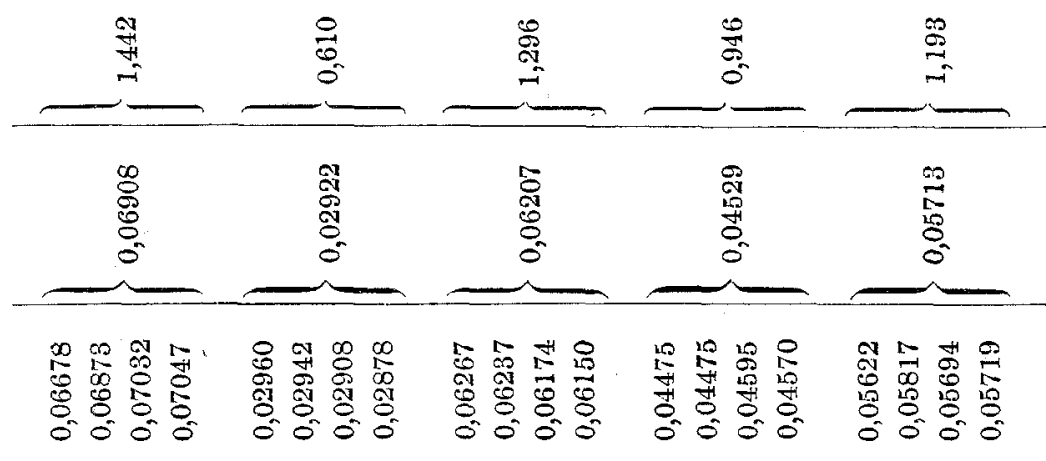

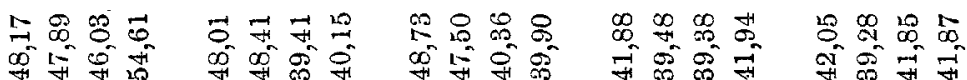

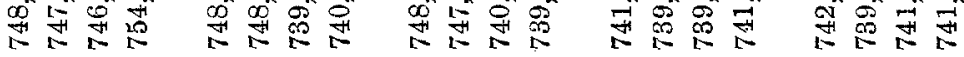

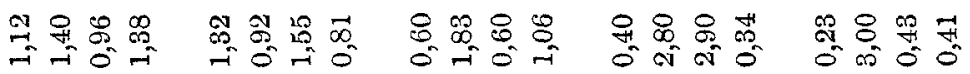

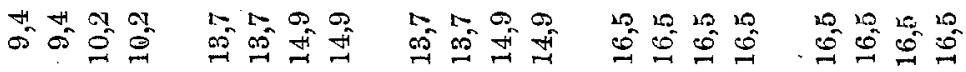

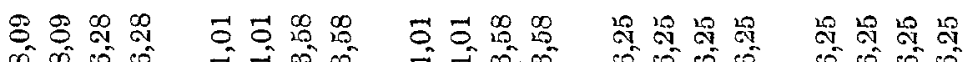
क

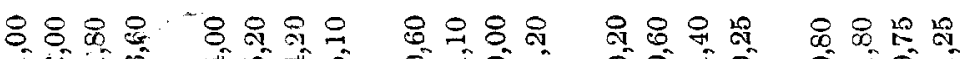

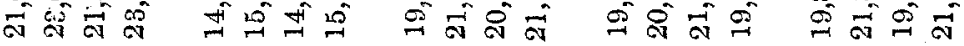

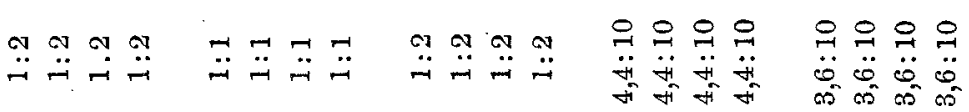

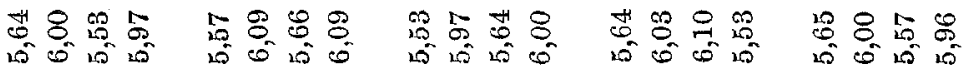

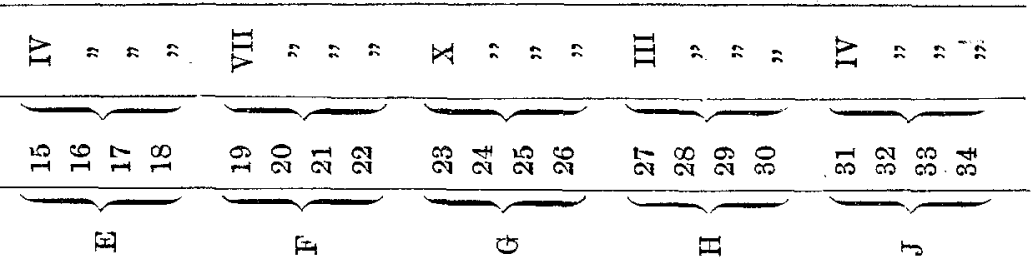


E. Pf」üger und K. Bohland:

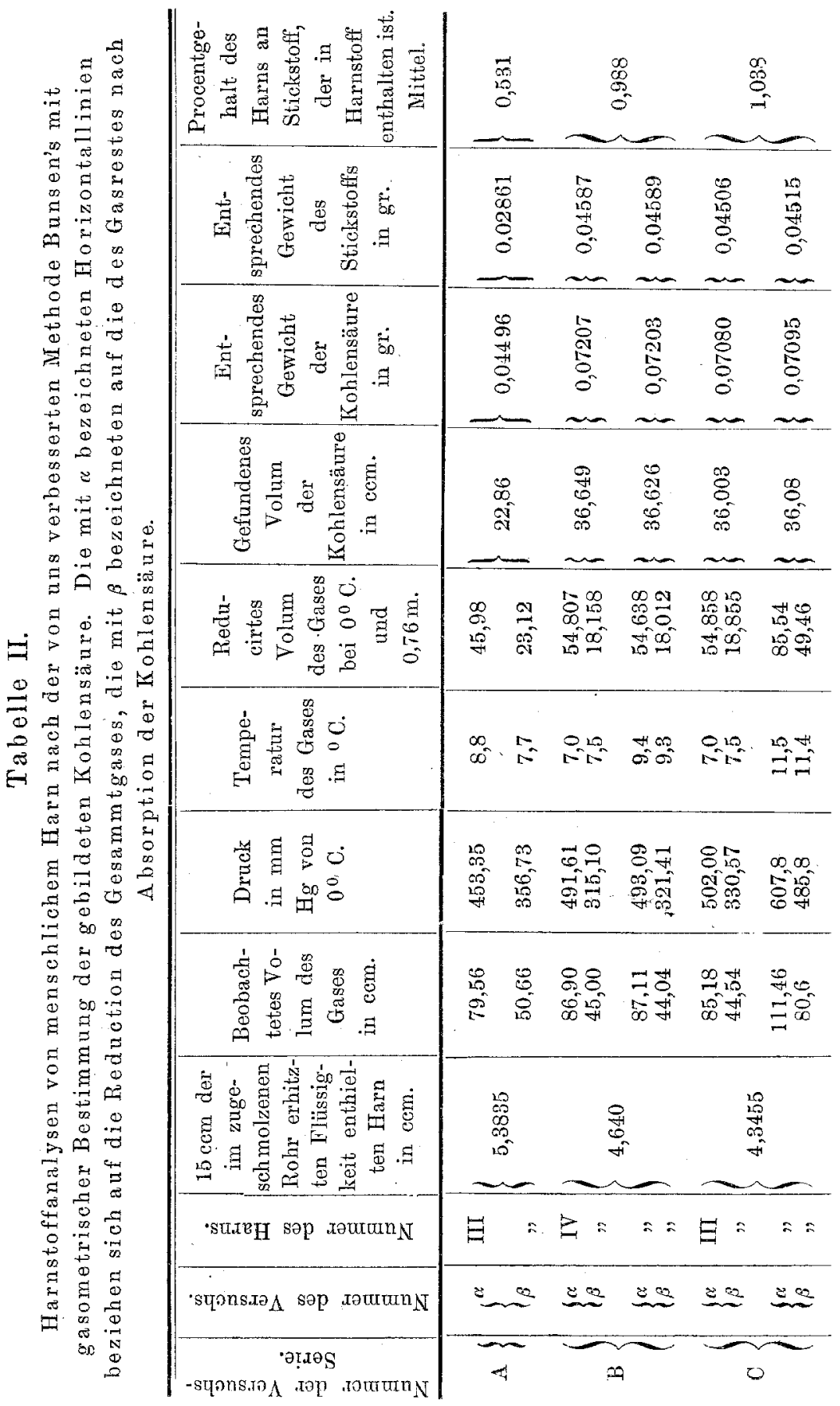




\begin{tabular}{|c|c|c|c|c|c|c|c|c|c|}
\hline & & & & & & & & $\begin{array}{l}\infty \\
\infty \\
\infty \\
0 \\
0\end{array}$ & $\stackrel{H}{0}$ \\
\hline $\begin{array}{l}8 \\
8 \\
0 \\
0\end{array}$ & \begin{tabular}{l}
$\infty$ \\
\multirow{2}{*}{} \\
9 \\
0 \\
0
\end{tabular} & $\begin{array}{l}19 \\
\text { f } \\
5 \\
0 \\
0 \\
0\end{array}$ & $\begin{array}{l}4 \\
0 \\
0 \\
8 \\
0 \\
0\end{array}$ & 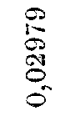 & $\begin{array}{l}8 \\
8 \\
8 \\
8\end{array}$ & $\begin{array}{l}5 \\
8 \\
0 \\
0 \\
0\end{array}$ & $\begin{array}{l}\infty \\
07 \\
0 \\
0 \\
0 \\
0\end{array}$ & $\begin{array}{l}0 \\
\frac{1}{1} \\
0 \\
0 \\
0\end{array}$ & $\begin{array}{l}\not{D} \\
\stackrel{0}{0} \\
0 \\
0\end{array}$ \\
\hline$m$ & $\approx$ & $m$ & $\sim$ & $m$ & $m$ & 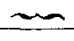 & $\sim$ & -1 & $m$ \\
\hline $\begin{array}{l}\infty \\
\stackrel{\infty}{0} \\
0 \\
0 \\
0\end{array}$ & 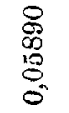 & $\begin{array}{l}7 \\
70 \\
0 \\
0 \\
0 \\
0\end{array}$ & $\begin{array}{l}0 \\
\frac{8}{46} \\
20 \\
0 \\
0 \\
0\end{array}$ & $\begin{array}{l}0 \\
0 \\
0 \\
0 \\
0 \\
0\end{array}$ & \begin{tabular}{l}
$\mathscr{c}$ \\
\multirow{2}{*}{} \\
0 \\
0
\end{tabular} & 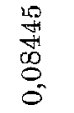 & \begin{tabular}{l}
$\infty$ \\
\multirow{2}{*}{} \\
$\infty$ \\
0 \\
0 \\
0
\end{tabular} & $\begin{array}{l}0 \\
\stackrel{0}{0} \\
0 \\
0\end{array}$ & $\begin{array}{l}\frac{1}{20} \\
5 \\
0 \\
0\end{array}$ \\
\hline$\cdots$ & $\simeq$ & $\simeq$ & $m$ & $m$ & 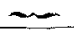 & $m$ & $\sim$ & 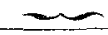 & $\sim$ \\
\hline 8 & 承 & 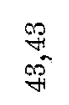 & 离 & 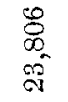 & 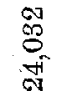 & 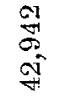 & $\begin{array}{l}\overrightarrow{7} \\
\vec{g}\end{array}$ & 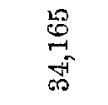 & 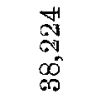 \\
\hline$\cdots$ & $m$ & $\cdots$ & $\cdots$ & $\cdots$ & $m$ & $\cdots$ & $\simeq$ & $\simeq$ & $\sim=$ \\
\hline 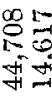 & $\begin{array}{l}1098 \\
080 \\
08 \\
392\end{array}$ & 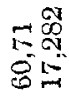 & $\begin{array}{l}+\infty \\
\sim \infty \\
\infty \\
0 \\
0\end{array}$ & 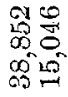 & 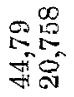 & $\begin{array}{l}95 \\
050 \\
005 \\
105=1\end{array}$ & $\begin{array}{l}80 \\
80 \\
80 \\
100 \\
100\end{array}$ & $\begin{array}{ll}10 & - \\
0 & \infty \\
0 & 0 \\
10 & 0\end{array}$ & 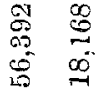 \\
\hline
\end{tabular}

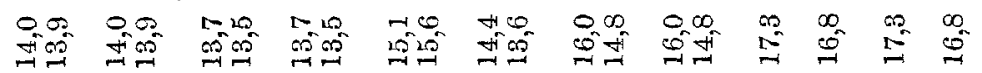

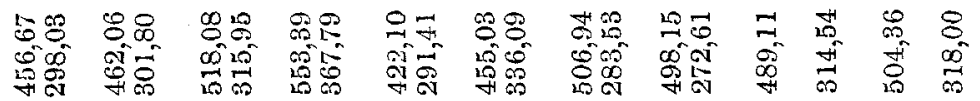

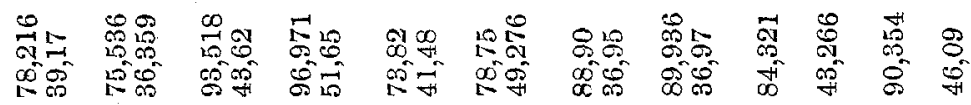

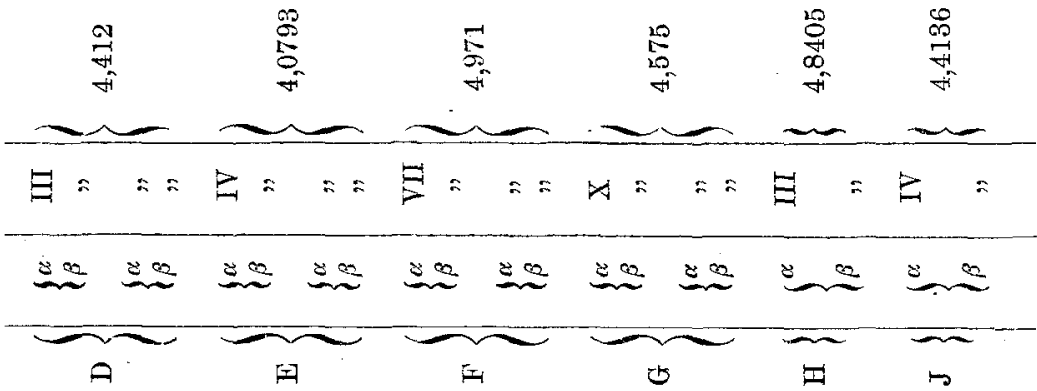


Tabelle III.

\begin{tabular}{|c|c|c|c|c|c|}
\hline 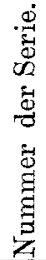 & $\begin{array}{c}\text { Procent- } \\
\text { gehalt an } \\
\text { Gesammt- } \\
\text { stickstoff } \\
\text { nach } \\
\text { Kjeldahl. }\end{array}$ & $\begin{array}{l}\text { Procentgehalt } \\
\text { an in Harnstoff } \\
\text { enthaltenem } \\
\text { Stickstoff } \\
\text { nach H ü fner. }\end{array}$ & $\begin{array}{l}\text { Procentgehalt } \\
\text { an in Harnstoff } \\
\text { enthaltenem } \\
\text { Stickstoff } \\
\text { nach Bunsen. }\end{array}$ & $\begin{array}{l}\text { Fehler von } \\
\text { Methode } \\
\text { Harnstoff. }\end{array}$ & $\begin{array}{l}\text { Hüfner's } \\
\text { ezogen auf } \\
\text { Gesammt- } \\
\text { stickstoff. }\end{array}$ \\
\hline A & 0,588 & 0,533 & 0,531 & $+\quad 0,4 \%$ & $-9,3 \%$ \\
\hline$B$ & 1,148 & 1,031 & 0,988 & $+4,3 n$ & $-10,2$ \\
\hline $\mathrm{C}$ & 1,178 & 1,056 & 1,038 & $+1,7$ & $-10,4$ \\
\hline $\mathrm{D}$ & 0,989 & 0,909 & 0,851 & $+6,8 \%$ & - 8,1 \\
\hline $\mathrm{E}$ & 1,568 & 1,442 & 1,332 & $+8,2 n$ & - 8,0 \\
\hline $\mathrm{F}$ & 0,676 & 0,610 & 0,603 & $+1,1 \%$ & $-\quad 9,7$, \\
\hline$G$ & 1,3996 & 1,296 & 1,178 & $+10,0$ & $-7,4$, \\
\hline $\mathrm{H}$ & - & 0,946 & 0,883 & $+7,1$ & - \\
\hline$J$ & - & 1,193 & 1,084 & $+10,1$ & - \\
\hline
\end{tabular}

Unsere Untersuchung hat also ergeben, dass Hü ner's Methode gerade so wie die von $\mathrm{Bunsen}$ zuweilen recht hefriedigende, ja gute Resultate gibt. Das ist aber auch hier nicht die Regel, sondern je nach der wechselnden Beschaffenheit des Harns ändert sich der Beobachtungsfehler und erreicht eventuell den bedentenden Werth von $+10 \%$. Ausnahmslos aber bleibt er positiv.

Wie die Untersuchung Pflüger's und Schenck's bewies, ist der Beobachtungsfehler stets negativ und schwankt übrigens fast in denselben Grenzen wie der positive, wenn man den nach Hüfner gewonnenen Werth mit dem Gesammtstickstoff vergleicht ${ }^{1}$ ).

Die Methode Hüfner's kann demnach weder zur Bestimmung des Gesammtstickstoffs noch zu der des Harnstoffs in Anwendung kommen, wenn es sich um Analysen handelt, bei denen Fehler bis zu $10 \%$ des gesuchten Werthes ausgeschlossen sein sollen. .

1) Siehe Dies Archiv XXXVIII, p. 334 u. 335. 


\title{
Analytische Belege.
}

\author{
Versuchserie $\mathrm{A}$.
}

\section{Harn III.}

I. Bestimmung des Gesammtstickstoffs nach Kjeldahl.

$75 \mathrm{ccm}$ Harn mit $40 \mathrm{ccm}$ rauchender Schwefelsäure 10 Stunden gekocht. Destiliationsvorlage: $29 \mathrm{ccm} 1 / 10$ Schwefelsäure.

Gebraucht $1 / 10$ Hyposulfitlösung $8,11 \mathrm{ccm}$ und $8,00 \mathrm{ccm}$, entsprechend: $0,02933 \mathrm{gr}$ und $0,02948 \mathrm{gr}$ Stickstoff;

Mittel: $0,02941 \mathrm{gr}$ Stickstoff.

Harn enthält also $0,588 \%$ Stickstoff in toto.

II. Bestimmung des Harnstoffs nach Bunsen in dem Harne, nachdem durch Phosphorwolframsäure, Salzsäure und nachherige Neutralisation die Extractivstoffe ausgefält sind.

$$
\begin{array}{cll}
200,38 \mathrm{~cm} & \text { Harn } \\
20,00 \quad \# & \text { Salzsäure } \\
60,00 \# & \text { Phosphorwolframsäure } \\
280,38 \mathrm{~cm} & \text { Mischung. }
\end{array}
$$

Controlversuch ergibt, dass das Volum der Mischung gleich der Summe der Volumina der einzelnen Bestandtheile. Es ist bei der Correctur also nur das Volum des Niederschlags zu berücksichtigen, welcher $0,27 \mathrm{ccm}$ ist. Wo bei der Beschreibung der folgenden Versuche analoge Verhältnisse obwalten, wird einfach das gemessene Volum des Niederschlags angegeben und in Rechnung gestellt. $100 \mathrm{ccm}$ dieser Mischung enthalten also 71,52 ccm Harn. Vom saneren Filtrat werden $200,21 \mathrm{~cm}=143,32 \mathrm{~cm}$ Harn abgemessen und mit Kalkpulver $\left(\mathrm{Ca}(\mathrm{OH})_{2}\right)$ zerrieben, d. h. alkalisch gemacht. Das durch besonderen Versuch festgestellte Volum des Niederschlags $=0,6 \mathrm{ccm}$. Folglich enthalten $5 \mathrm{ccm}$ des Filtrates jetzt 3,589 ccm Harn.

Das durch $\mathrm{Ca}(\mathrm{OH})_{2}$ alkalische Filtrat wird mit gleichem Volumen alkalischer $\mathrm{BaCl}_{2}$-Lösung versetzt, je $15 \mathrm{ccm}$ Filtrat eingeschmolzen und 4 Stunden auf $220^{\circ}$ erhitzt.

Bestimmung dergebildeten Kohlensäure.

Ablesung am Absorptionsrohr:

$$
\begin{aligned}
& \mathrm{Hgu}=66,65 \\
& \text { Hgo }=37,02 \quad \text { Kaliber }\left\{\begin{array}{l}
37,79 \mathrm{~cm}=81,0 \mathrm{~cm} \quad \mathrm{~T}=8,8^{0} \mathrm{C} . \\
1 \mathrm{~cm}=2,158 \mathrm{~cm} . \quad \mathrm{Ba}=758,8 \mathrm{~mm} .
\end{array}\right. \\
& \mathrm{V}=79,56 \mathrm{~cm} ; \mathrm{P}=453,35 \mathrm{~mm} . \\
& \text { Reducirtes Gesammtgas }=45,98 \mathrm{ccm} .
\end{aligned}
$$


Nach Absorption der Kohlensäure:

Ablesungen am Absorptionsrohr:

$$
\begin{aligned}
& \text { Hgu }=66,75 \\
& \text { Hgo }=27,35 \\
& \mathrm{KOH}=23,69
\end{aligned} \quad \text { Kaliber }\left\{\begin{array}{l}
23,85 \mathrm{~cm}=51,0 \mathrm{~cm} \quad \mathrm{~T}=7,7^{\circ} \mathrm{C} . \\
1 \mathrm{~cm}=2,14 \mathrm{~cm} . \quad \mathrm{Ba}=758,2 \mathrm{~mm} .
\end{array}\right.
$$

Folglich $22,86 \mathrm{ccm} \mathrm{CO}_{2}=44,96 \mathrm{mgr} \mathrm{CO} \mathrm{C}_{2}=61,31 \mathrm{mgr} \stackrel{+}{\mathrm{U}}=0,02861 \mathrm{gr}$ Stickstoff. Demnach $5 \mathrm{ccm}$ Harn $=0,02657 \mathrm{gr}$ Stickstoff oder der Harn enthält in Harnstoff $0,531 \%$ Stickstoff.

\section{Versuchserie B.}

\section{Harn IV.}

\section{Gesammtstickstoff.}

Je $5 \mathrm{ccm}$ Harn mit $40 \mathrm{ccm}$ rauchender Schwefelsäure 10 Stunden gekocht. Vorlage am Destillirapparat: $47 \mathrm{ccm} \quad 1 / 10 \quad \mathrm{SO}_{4} \mathrm{H}_{2}$.

$1 / 10$ Hypostifit-lösung $6,17 \mathrm{ccm} ; 6,04 \mathrm{ccm} ; 6,13 \mathrm{ccm}$; entsprechend Stickstoff $0,05733 \mathrm{gr} ; 0,05751 \mathrm{gr} ; 0,05739 \mathrm{gr}$.

Mittel: $0,5741 \mathrm{gr}$ Stickstoff.

Der Harn enthält: 1,148\% Stickstoff in toto.

II. Harnstoffbestimmung nach Bunsen nach Entfernung der durch Phosphorwolframsäure fällbaren Extractivstoffe.

$$
\begin{aligned}
& \text { 200,26 com Harn } \\
& 20,00 \text { "Salzsäure } \\
& 100,00^{\circ} " \text { Phosphorwolframsäure } \\
& 320,26 \mathrm{~cm} \text { 'Mischung. }
\end{aligned}
$$

Das Volum des entstandenen Niederschlags betrug $0,76 \mathrm{~cm}$.

$200,26 \mathrm{~cm}$. des Filtrates $=125,5 \mathrm{ccm}$ Harn wurden mit Kalkpulver $\left(\mathrm{Ca}(\mathrm{OH})_{2}\right)$ zerrieben und alkalisch gemacht. Bei der Ausfällung mit $\mathrm{Ca}(\mathrm{OH})_{2}$ fand eine Expansion ron $4,2 \mathrm{ccm}$ statt, das Volum des Niederschlags betrug 1,5 ccm, sodass ein Plus von 2,7 ccm Expansion bleibt. Das Filtrat wurde mit gleichem Volum alkalischer $\mathrm{BaCl}_{2}$-Lösung versetzt und jo $15 \mathrm{ccm}$ eingeschmolzen. $100 \mathrm{~cm}$ dieser Mischung $=30,915 \mathrm{ccm}$ Harn. $15 \mathrm{ccm}=4,64 \mathrm{ccm}$. (Uncorrigirt: $4,69 \mathrm{~cm}$.)

Kohlensäurebestimmung.

Rohr I.

$$
\begin{aligned}
& \begin{array}{l}
\mathrm{Hgu}=66,36 \\
\mathrm{Hgo}=40,35
\end{array} \quad \text { Kaliber } \begin{cases}40,50 \mathrm{~cm}=87 \mathrm{ccm} & \mathrm{T}=7,0^{\circ} \mathrm{C} . \\
1 \mathrm{~cm}=2,22 \mathrm{~cm} & \mathrm{Ba}=759,8 \mathrm{~mm} .\end{cases} \\
& \mathrm{V}=86,90 ; \mathrm{P}=491,61 \mathrm{~mm} \text {. } \\
& \text { Reducirtes Gesammtgas }=54,807 \mathrm{ccm} \text {. }
\end{aligned}
$$


Nach Absorption der Kohlensäure:

$$
\begin{array}{ll}
\mathrm{Hgu}=66,62 \\
\mathrm{Hgo}=22,65 \\
\mathrm{KOH}=21,05
\end{array} \quad \text { Kaliber } \begin{cases}21,05 \mathrm{~cm}=45 \mathrm{~cm} & \mathrm{~T}=7,5^{0} \mathrm{C} . \\
& \mathrm{Ba}=760,05 \mathrm{~mm} .\end{cases}
$$

$\mathrm{V}=45,0 \mathrm{ccm} ; \quad \mathrm{P}=315,10 \mathrm{~mm}$.

Reducirtes restirendes Gas $=18,158 \mathrm{~cm}$.

Folglich: $\quad 36,649 \mathrm{ccm} \quad \mathrm{CO}_{2}=72,074 \mathrm{mgr}=98,28 \mathrm{mgr} \quad \stackrel{+}{\mathrm{U}}=0,04587 \mathrm{gr}$ Stickstoff.

Also $5 \mathrm{ccm}$ Harn $=0,04943 \mathrm{gr}$ Stickstoff in Harnstoff.

Rohr II.

$$
\begin{aligned}
& \begin{array}{l}
\mathrm{Hgu}==66,11 \\
\mathrm{Hgo}=40,45
\end{array} \quad \text { Kaliber } \begin{cases}40,50=87 \mathrm{~cm} & \mathrm{~T}=9,40 \mathrm{C} . \\
1 \mathrm{~cm}=2,22 \mathrm{ccm} & \mathrm{Ba}=759,31 \mathrm{~mm} .\end{cases} \\
& \mathrm{V}=87,11 \mathrm{ccm} ; \quad \mathrm{P}=493,09 \mathrm{~mm} \text {. } \\
& \text { Reducirtes Gesammtgas }=54,638 \mathrm{ccm} \text {. }
\end{aligned}
$$

Nach Absorption der Kohlensäure:

$$
\begin{aligned}
& \text { Hgu }=66,37 \\
& \mathrm{Hgo}=23,31 \\
& \mathrm{KOH}=20,60
\end{aligned} \quad \text { Kaliber } \begin{cases}21,05 \mathrm{~cm}=45 \mathrm{~cm} & \mathrm{~K}=9,30 \mathrm{c} . \\
1 \mathrm{~cm}=2,13 \mathrm{~cm} & \mathrm{Ba}=758,9 \mathrm{~mm} . \\
\mathrm{V}=44,04 \mathrm{~cm} ; \mathrm{P}=321,41 \mathrm{~mm} . \\
\text { Reducirtes restirendes Gas }=18,012 .\end{cases}
$$

Folglich : $36,626 \mathrm{ccm} \mathrm{CO}_{2}=72,03 \mathrm{mgr} \mathrm{CO}_{2}=98,223 \mathrm{mgr} \stackrel{+}{U}=0,04589 \mathrm{gr}$ Stickstoff.

$5 \mathrm{ccm}$ Harn also $=0,04940 \mathrm{gr}$ Stickstoff.

$5 \mathrm{ccm}$ Harn im Mittel also $=0,04942 \mathrm{gr}$ Stickstoff in Harnstoff.

Der Harn enthält also $0,988 \%$ " " "

\section{Versuchsserie C.}

\section{Harn III.}

I. Gesammtstickstoff.

Je $5 \mathrm{~cm}$ Harn mit $40 \mathrm{~cm}$ rauchender Schwefelsäure 10 Stunden gekocht. Vorlage $47 \mathrm{ccm} 1 / 10 \mathrm{SO}_{4} \mathrm{H}_{2}$.

1/10 Hyposulfitlösung: $5,09 \mathrm{com}$ and $5,03 \mathrm{ccm}$;

entsprechend: $0,05884 \mathrm{gr}$ und $0,05893 \mathrm{gr}$ Stickstoff;

Mittel $=0,5888 \mathrm{gr}$ Stickstoff;

Harn enthält: $1,1777 \%$ Stickstoff in toto.

II. Harnstolfbestimmung nach Bunsen mit Elimination der durch Phosphorwolframsäure fällbaren "Extractivstoffe".

$$
\begin{aligned}
& \text { 200,38 ecm Harn } \\
& \text { 20,00 "Salzsäure } \\
& \text { 120,00 " Phosphorwolframsäure } \\
& \text { 340,38 ccm Mischung. }
\end{aligned}
$$


Volum des entstandenen Niederschlages $=0,82 \mathrm{ccm} .200,38 \mathrm{ccm}$ des Filtrates $=118,244 \mathrm{ccm}$ Harn und Kalkpulver alkalisch gemacht, das Filtrat mit gleichem Volum alkalischer Chlorbariumlösung verzetzt und von dem Filtrat hiervon je $15 \mathrm{ccm}$ eingeschmolzen. Bei der Ausfällung mit $\mathrm{Ca}(\mathrm{OH})_{2}$ betrug die Expansion 5,7 ccm; das Volum des Niederschlags 2,0 ccm, folglich Plus an Expansion $=3,7 \mathrm{ccm}, 15 \mathrm{ccm}$ der alkalischen $\mathrm{BaCl}_{2}$-Harnmischung also $=4,3455 \mathrm{ccm}$ Harn.

Kohlensäur ebestimmung.

Rohr I.

$$
\begin{aligned}
& \begin{array}{l}
\text { Hgu }=64,97 \\
\text { Hgo }=40,00
\end{array} \quad \text { Kaliber } \begin{cases}40,95 \mathrm{~cm}=87 \mathrm{ccm} & \mathrm{T}=7,0^{\circ} \mathrm{C} . \\
1 \mathrm{~cm}=2,143 \mathrm{ccm} & \mathrm{Ba}=759,8 \mathrm{~mm} .\end{cases} \\
& \mathrm{V}=85,18 \mathrm{ccm} ; \quad \mathbf{P}=502,00 \mathrm{~mm} \text {. }
\end{aligned}
$$

Reducirtes Gesammtgas $=54,858 \mathrm{ccm}$.

Nach Absorption der Kohlensäure:

$$
\begin{aligned}
& \begin{array}{l}
\text { Hgu }=65,25 \\
\text { Hgo }=22,85 \\
\text { KOH }=21,05
\end{array} \quad \text { Kaliber } \begin{cases}21,27 \mathrm{~cm}=45 \mathrm{~cm} & \mathrm{~T}=7,5^{0} \mathrm{C} . \\
1 \mathrm{~cm}=2,11 \mathrm{~cm} & \mathrm{Ba}=760,5 \mathrm{~mm} .\end{cases} \\
& \mathrm{V}=44,54 \mathrm{ccm} ; \quad \mathrm{P}=330,57 \text {. }
\end{aligned}
$$
Stickstoff.

Folglich : $36,003 \mathrm{ccm} \mathrm{CO}_{2}=70,804 \mathrm{mgr} \mathrm{CO}_{2}=96,55 \mathrm{mgr} \stackrel{+}{\mathrm{U}}=0,04506 \mathrm{gr}$

$$
5 \mathrm{ccm} \text { Harn also }=0,05185 \mathrm{gr} \text { Stickstoff. }
$$

Rohr II.

$$
\begin{gathered}
\text { Hgu }=65,96 \\
\text { Hgo }=51,56
\end{gathered} \text { Kaliber } \begin{cases}51,45 \mathrm{~cm}=111 \mathrm{~cm} & \mathrm{~T}=11,5^{\circ} \mathrm{C} . \\
1 \mathrm{~cm}=2,22 \mathrm{~cm} & \mathrm{Ba}=763,1 \mathrm{~mm} .\end{cases}
$$

Reducirtes Gesammtgas $=85,54 \mathrm{ccm}$.

Nach Absorption der Kohlensäure:

$$
\begin{aligned}
& \begin{array}{l}
\text { Hgu }=66,05 \\
\mathrm{Hgo}=39,06 \\
\mathrm{KOH}=37,60
\end{array} \quad \text { Kaliber } \begin{cases}37,79 \mathrm{~cm}=81 \mathrm{~cm} & \mathrm{~T}=11,4^{0} \mathrm{C} . \\
1 \mathrm{~cm}=2,158 \mathrm{ccm} & \mathrm{Ba}=762,3 \mathrm{~mm} .\end{cases} \\
& \mathrm{V}=80,6 \mathrm{ccm} ; \quad \mathrm{P}=485,8 \mathrm{~mm} \text {. }
\end{aligned}
$$

Folglich : $36,08 \mathrm{~cm} \mathrm{CO}_{2}=70,955 \mathrm{mgr} \mathrm{CO} 2=96,76 \mathrm{mgr} \stackrel{+}{\mathrm{U}}=0,04515 \mathrm{gr}$ Stickstoff.

$$
\begin{aligned}
5 \mathrm{ccm} \text { also } & =0,05192 \mathrm{gr} \text { Stickstoff: } \\
\text { Mittel } & =0,05188 \mathrm{gr} \text { Stickstoff. }
\end{aligned}
$$

Dẹr Hạn enthält also 1,038\% Stickstoff an Harnstoff gebunden. 


\section{Versuchsserie D.}

\section{Harn III.}

I. Gesammtstickstoff.

Je $5 \mathrm{ccm}$ Harn mit $40 \mathrm{ccm}$ rauchender Schwefelsäure 10 Stunden gekocht. Vorlage $47 \mathrm{ccm} 1 / 10 \mathrm{SO}_{4} \mathrm{H}_{2}$.

$1 / 10$ Hyposulfitlösung $=11,75 \mathrm{ccm}$ und $11,82 \mathrm{ccm}$, entsprechend: $0,04949 \mathrm{gr}$ und $0,04939 \mathrm{gr}$ Stickstoff.

$$
\text { Mittel }=0,04944 \mathrm{gr} \text { Stickstof: }
$$

Harn enthält also: $0,989 \%$ Stickstoff in toto.

II. Harnstoffbestimmung nach Bunsen nach Elimination an durch Phosphorwolframsäure fällbaren "Extractivstoffe".

$$
\begin{aligned}
& 200,21 \mathrm{~cm} \text { Harn } \\
& 20,00 \Rightarrow \text { Salzsäure } \\
& 120,00 " \text { Phosphorwolframsäure } \\
& \hline 340,21 \mathrm{ccm} \text { Mischung. }
\end{aligned}
$$

Volum des entstandenen Niederschlags $=1,4 \mathrm{ccm} .-200,1 \mathrm{ccm}$ des Filtrats $=118,243 \mathrm{ccm}$ Harn mit Kalkpulver $\left(\mathrm{Ca}(\mathrm{OH})_{2}\right)$ alkalisch gemacht; das Filtrat mit gleichem Volum alkalischer $\mathrm{BaCl}_{2}$-Lösung versetzt und filtrirt. Je $15 \mathrm{ccm}=4,412 \mathrm{ccm}$ Harn eingeschmolzen. Expansion bei Ausfällung mit $\mathrm{Ca}(\mathrm{OH})_{2}$ betrug $4,87 \mathrm{ccm}$; Volum des entstandenen Niederschlags $3,97 \mathrm{ccm}$; folglich ein Plus an Expansion von 0,9 ccm.

Kohlensäurebestimmung.

Rohr I.

$$
\begin{aligned}
& \begin{aligned}
\mathrm{Hgu}=65,49 \\
\mathrm{Hgo}=36,40
\end{aligned} \quad \text { Kaliber }\left\{36,4 \mathrm{~cm}=78 \mathrm{~cm} \begin{array}{rl}
\mathrm{T} & =14,0^{0} \mathrm{C} . \\
\mathrm{Ba} & =760,6 \mathrm{~mm} .
\end{array}\right. \\
& \mathrm{V}=78,216 \mathrm{ccm} ; \quad \mathrm{P}=456,67 \mathrm{~mm} \text {. } \\
& \text { Reducirtes Gesammtgas }=44,708 \mathrm{ccm} \text {. }
\end{aligned}
$$

Nach Absorption der Kohlensäure:

$$
\begin{aligned}
& \mathrm{Hgu}=65,76 \\
& \text { Hgo }=20,11 \quad \text { Kaliber }\left\{18,25 \mathrm{~cm}=39 \mathrm{~cm} \quad \mathrm{~T}=13,9^{0} \mathrm{C}\right.
\end{aligned}
$$

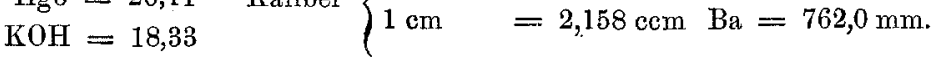

$$
\begin{aligned}
& \mathrm{V}=39,17 \mathrm{ccm} ; \quad \mathrm{P}=298,03 \mathrm{~mm} \text {. } \\
& \text { Reducirtes restirendes Gas }=14,617 \mathrm{ccm} \text {. }
\end{aligned}
$$

Stickstoff.

Folglich $30,09 \mathrm{~cm} \mathrm{CO}_{2}=59,18 \mathrm{mgr} \quad \mathrm{CO}_{2}=80,70 \mathrm{mgr} \stackrel{+}{\mathrm{U}}=0,03766 \mathrm{gr}$

Rohr II.

$$
\begin{aligned}
& \begin{array}{l}
\text { Hgu }=64,05 \\
\text { Hgo }=35,50
\end{array} \text { Kaliber }\left\{\begin{array}{l}
35,35 \mathrm{~cm}=75 \mathrm{~cm} \quad \mathrm{~T}=14,0^{0} \mathrm{C} . \\
1 \mathrm{~cm}=2,143 \mathrm{ccm} \mathrm{Ba}=760,6 \mathrm{~mm} .
\end{array}\right. \\
& \mathrm{V}=75,536 \mathrm{ccm} ; \quad \mathrm{P}=462,06 \mathrm{~mm} \text {. } \\
& \text { Reducirtes Gesammtgas }=43,685 \mathrm{ccm} \text {. }
\end{aligned}
$$


Nach Absorption der Kohlensäure:

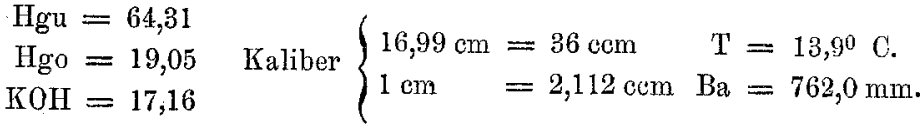

$$
\begin{aligned}
& \mathrm{V}=36,359 \mathrm{ccm} ; \quad \mathrm{P}=301,8 \mathrm{~mm} \text {. } \\
& \text { Reducirtes restirendes Gas }=13,739 \mathrm{ccm} \text {. }
\end{aligned}
$$
Stickstoff.

Folglich $29,95 \mathrm{ccm} \mathrm{CO}_{2}=58,90 \mathrm{mgr} \quad \mathrm{CO}_{2}=80,32 \mathrm{mgr} \stackrel{+}{\mathrm{U}}=0,03748 \mathrm{gr}$

$$
\begin{gathered}
\text { Mittel }=0,03757 \mathrm{gr}^{*} \text { Stickstoff. } \\
5 \mathrm{ccm} \text { Harn }=0,04257 \mathrm{gr} \text { Stickstoff. } \\
\text { Der Harn enthält: } 0,851 \% \text { Stickstoff in Harnstoff. }
\end{gathered}
$$

\section{Versuchsserie E.}

\section{Harn IV.}

\section{Gesammtstickstoff} kocht.

Je $5 \mathrm{ccm}$ Harn mit $40 \mathrm{ccm}$ rauchender Schwefelsäure 10 Stunden geVorlage: $66 \mathrm{ccm} 1 / 10 \mathrm{SO}_{4} \mathrm{H}_{2}$ :

$1 /$ 10. Hyposulfitlösung $=10,19 \mathrm{ccm}$ and $10,13 \mathrm{ccm}$ entsprechend: $0,07836 \mathrm{gr}$ und $0,7844 \mathrm{gr}$ Stickstoff.

Mittel: $0,07840 \mathrm{gr}$ Stickstoff.

Harn enthält also: $1,568 \%$ Stickstoff in toto.

II. Harnstoffbestimmung nach Bunsen nach Elimination der durch Phosphorwolframsäure fällbaren "Extractivstoffe".

$$
\begin{aligned}
& 200,1 \mathrm{ccm} \text { Harn } \\
& 20,0 \text { " Salzsäure } \\
& 150,0 \text { " Phosphorwolframsäure } \\
& \text { 370,1 ccm Mischung. }
\end{aligned}
$$

Volum des entstándenen Niederschlages $=2,2 \mathrm{ccm} .-200,21 \mathrm{ccm}$ des Filtrats $=108,894$ Harn mit Kalkpulver $\left(\mathrm{Ca}(\mathrm{OH})_{2}\right)$ alkalisch gemacht; das Filtrat mit gleichem Volum alkalischer $\mathrm{Cl}_{2} \mathrm{Ba}$-Lösung versetzt und von diesem Filtrat je $15 \mathrm{ccm}=4,0793 \mathrm{~cm}$ Harn eingesehmolzen. Bei der Ausfällung mit $\mathrm{Ca}(\mathrm{OH})_{2}$ hatte die Expansion $4,32 \mathrm{ccm}$ betragen, das Volum des Niederschlags ebenfalls $4,32 \mathrm{gcm}$.

Kohlensäurebestimmung.

Rohr I.

$$
\begin{aligned}
& \begin{array}{l}
\text { Hgu }=66,15 \\
\text { Hgo }=43,39
\end{array} \text { Kaliber } \begin{cases}43,25 \mathrm{~cm}=93 \mathrm{ccm} & \mathrm{T}=13,7^{0} \mathrm{C} . \\
1 \mathrm{~cm} \quad=2,158 \mathrm{~cm} & \mathrm{Ba}=758,6 \mathrm{~mm} .\end{cases} \\
& \mathrm{V}=93,518 \mathrm{ccm} ; \quad \mathbf{P}=518,08 \mathrm{~mm} \text {. } \\
& \text { Reducirtes Gesammtgas }=60,71 \mathrm{~cm} \text {. }
\end{aligned}
$$


Nach Absorption der Kohlensäure:

$$
\begin{gathered}
\mathrm{Hgu}=66,45 \\
\mathrm{HgO}=22,85 \\
\mathrm{KOH}=20,40 \\
\mathrm{~V}=43,62 \mathrm{ccm} ; \mathrm{P}=315,95 \mathrm{~mm} . \\
\text { Reducirtes restirendes Gas }=17,282 \mathrm{ccm} .
\end{gathered}
$$

Folglich $43,43 \mathrm{ccm} \mathrm{CO}_{2}=85,41 \mathrm{mgr} \mathrm{CO}_{2}=116,47 \mathrm{mgr} \stackrel{+}{\mathrm{U}}=0,05435 \mathrm{gr}$ Stickstoff.

Rohr II.

$$
\begin{aligned}
& \begin{array}{l}
\text { Hgu }=64,67 \\
\text { Hgo }=45,45
\end{array} \quad \text { Kaliber }\left\{\begin{array}{l}
45,1 \mathrm{~cm}=96 \mathrm{ccm} \\
1 \mathrm{~cm}=2,158 \mathrm{ccm} \quad \mathrm{Ba}=13,7^{0} \mathrm{C} . \\
=758,6 \mathrm{~mm} .
\end{array}\right. \\
& \mathrm{V}=96,971 \mathrm{~cm} ; \mathrm{P}=553,39 \mathrm{~mm} \text {. } \\
& \text { Reducirtes Gesammtgas }=67,24 \mathrm{ccm} \text {. }
\end{aligned}
$$

Nach Absorption der Kohlensäure:

$$
\begin{gathered}
\mathrm{Hgu}=64,97 \\
\mathrm{Hga}=26,53 \quad \text { Kaliber }\left\{\begin{array}{l}
24,11 \mathrm{~cm}=51 \mathrm{~cm} \quad \mathrm{~T}=13,5^{\circ} \mathrm{C} . \\
1 \mathrm{~cm}=2,112 \mathrm{ccm} \quad \mathrm{Ba}=760,0 \mathrm{~mm} .
\end{array}\right. \\
\mathrm{KOH}=24,42 \\
\quad \mathrm{~V}=51,65 \mathrm{ccm} ; \mathrm{P}=367,79 \mathrm{~mm} . \\
\text { Reducirtes restirendes Gas }=23,818 \mathrm{ccm} .
\end{gathered}
$$

Folglich 43,424 cem $\mathrm{CO}_{2}=85,398 \mathrm{mgr} \mathrm{CO}_{2}=116,45 \mathrm{mgr} \stackrel{+}{\mathrm{U}}=0,054344 \mathrm{gr}$ Stickstoff.

$$
\text { Mittel }=0,054345 \mathrm{gr} \text { Stickstoff. }
$$

$5 \mathrm{ccm}$ Harn $=0,06661 \mathrm{gr}$ Stickstoff.

Der Harn enthält also $1,332 \%$ Stickstoff in Harnstoff.

\section{Versuchsserie F.}

\section{Harn VII.}

I. Gesammtstickstoff.

Je $5 \mathrm{ccm}$ Harn mit $40 \mathrm{ccm}$ rauchender Schwefelsäure 10 Stunden gekocht. Vorlage $34 \mathrm{cem} 1 / 10 \quad \mathrm{SO}_{4} \mathrm{H}_{2}$

$1 / 10$ Hyposulfitlösung $=9,9 \mathrm{ccm}$ und $9,98 \mathrm{ccm}$, entsprechend: $0,03384 \mathrm{gr}$ und $0,03372 \mathrm{gr}$ Stickstoff;

Mittel $=0,03378 \mathrm{gr}$ Stickstoff.

Harn $=0,676 \%$ Stickstoff in toto.

II. Harnstoffbestimmung nach Bunsen nach Elimination der durch Phosphorwolframsäure fällbaren "Extractivstoffe".

$$
\begin{aligned}
& 200,29 \mathrm{ccm} \text { Harn } \\
& 20,00 \# \text { Salzsäure } \\
& 80,00 \# \text { Phorphorwolframsäure } \\
& \hline 300,29 \text { com. Mischung. }
\end{aligned}
$$


Volum des entstandenen Niederschlags $0,35 \mathrm{ccm}$.

$200,29 \mathrm{~cm}$ des Filtrates $=133,745 \mathrm{~cm}$ Harn mit Kalkpulver $\left[\mathrm{Ca}(\mathrm{OH})_{2}\right]$ alkalisch gemacht; das Filtrat mit gleichem Volum alkalischer $\mathrm{BaCl}_{2}$-Lösung versetzt und filtrirt; von dieser Mischung je $15 \mathrm{ccm}=4,971 \mathrm{ccm}$ Harn eingeschmolzen. Die Expansion bei der Ausfällung mit $\mathrm{Ca}(\mathrm{OH})_{2}$ hatte betragen $3,00 \mathrm{ccm}$; das Volum des entstandenen Niederschlages 1,5 com, so dass ein Plus an Expansion von 1,5 ecm bleibt.

Kohlensäurebestimmung.

Rohr I.

$$
\begin{gathered}
\text { Hgu }=66,70 \\
\text { Hgo }=34,35 \quad \text { Kaliber }\left\{\begin{array}{l}
33,60 \mathrm{~cm}=72 \mathrm{~cm} \quad \mathrm{~T}=15,1^{0} \mathrm{C} . \\
1 \mathrm{~cm}=2,142 \mathrm{ccm} \quad \mathrm{Ba}=759,5 \mathrm{~mm} .
\end{array}\right. \\
\mathrm{V}=73,82 \mathrm{~cm} ; \mathrm{P}=422,10 \mathrm{~mm} . \\
\text { Reducirtes Gesammtgas }=38,852 \mathrm{ccm} .
\end{gathered}
$$

Nach Absorption der Kohlensäure:

$$
\begin{gathered}
\mathrm{Hgu}=66,80 \\
\mathrm{Hgo}=20,76 \\
\mathrm{KOH}=19,40
\end{gathered} \quad \text { Kaliber }\left\{\begin{array}{l}
19,64 \mathrm{~cm}=42 \mathrm{ccm} \\
1 \mathrm{~cm}=2,158 \mathrm{~cm} \quad \mathrm{Ba}
\end{array}\right.
$$

Folglich $23,806 \mathrm{~cm} \mathrm{CO}_{2}=46,817 \mathrm{mgr}^{\mathrm{CO}} \mathrm{O}_{2}=63,84 \mathrm{mgr} \stackrel{+}{\mathrm{U}}=0,02979 \mathrm{gr}$ Stickstoff.

Rohr II.

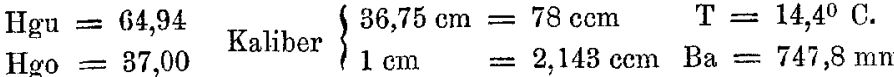

$$
\begin{aligned}
& \mathrm{V}=78,75 \mathrm{~cm} ; \quad \mathrm{P}=455,03 \mathrm{~mm} \text {. } \\
& \text { Reducirtes Gesammtgas }=44,79 \mathrm{ccm} \text {. }
\end{aligned}
$$

Nach Absorption der Kohlensäure:

$$
\begin{aligned}
& \begin{aligned}
\text { Hgu } & =65,13 \\
\text { Hgo } & =24,34 \\
\text { KOH } & =23,30
\end{aligned} \quad \text { Kaliber }\left\{\begin{array}{l}
24,11 \mathrm{~cm}=51 \mathrm{~cm} \quad \mathrm{~T}=13,6^{0} \mathrm{C} . \\
1 \mathrm{~cm}=2,128 \mathrm{ccm} \mathrm{Ba}=750,7 \mathrm{~mm} .
\end{array}\right. \\
& \mathrm{V}=49,276 \mathrm{~cm} ; \quad \mathrm{P}=336,09 \mathrm{~mm} \text {. } \\
& \text { Reducirtes restirendes Gas }=20,758 \mathrm{ccm} \text {. }
\end{aligned}
$$

Folglich $24,032 \mathrm{ccm} \mathrm{CO} 2=47,262 \mathrm{mgr} \mathrm{CO}_{2}=64,447 \mathrm{mgr} \stackrel{+}{\mathrm{U}}=0,03007 \mathrm{gr}$ Stickstoff.

$$
\text { Mittel }=0,02993 \mathrm{gr} \text { Stickstoff. }
$$

$5 \mathrm{ccm}$ Harn enthalten: $0,03014 \mathrm{gr}$ Stickstoff in Harnstoff.

Der Harn enthält: $0,603 \%$ Stickstoff in Harnstoff. 


\section{Versuchsserie G.}

\section{Harn X.}

\section{Gesammtstickstoff.}

Je $5 \mathrm{ccm}$ Harn mit $40 \mathrm{ccm}$ rauchender Schwefelsäure 10 Stunden gekocht. Vorlage $55 \mathrm{ccm} 1 / 10 \quad \mathrm{SO}_{4} \mathrm{H}_{2}$.

$1 / 10$ Hyposulfitlösung $5,10 \mathrm{~cm}$ und $5,22 \mathrm{ccm}$, entsprechend: 0,07006 gr und $0,06989 \mathrm{gr}$ Stickstoff.

Mittel: $0,06998 \mathrm{gr}$ Stickstoff.

Harn enthält 1,3996\% Stickstoff in toto.

I. Harnstoffbestimmung nach Bunsen nach Elimination der durch Phosphorwolframsäure fällbaren "Extractivstoffe."

$$
\begin{aligned}
& 200,18 \mathrm{ccm} \text { Harn } \\
& 20,00 \# \text { Salzsäure } \\
& 110,00 \# \text { Phosphorwolframsäure } \\
& 330,18 \mathrm{ccm} \text { Mischung. }
\end{aligned}
$$

Volum des entstandenen Niederschlages $=1,65 \mathrm{ccm} .200,18 \mathrm{ccm}$ des sauren Filtrates $=121,97 \mathrm{~cm}$ Harn mit Kalkpulver $\left[\mathrm{Ca}(\mathrm{OH})_{2}\right]$ alkalisch gemacht; das Filtrat mit gleichem Volum alkalischer $\mathrm{BaCl}_{2}$-lösung versetzt und filtrirt. Von dieser Mischung je $15 \mathrm{~cm}$ eingeschmolzen.

Die Expansion bei Ausfällung mit $\mathrm{Ca}(\mathrm{OH})_{2}$ betrug $3,52 \mathrm{ccm}$.

Das Volum des entstandenen Niederschlages betrug 3,30 "

Folglich bleibt ein Plus an Expansion $=0,22$,

$15 \mathrm{ccm}$ der eingeschmolzenen Mischung also 4,575 ccm Harn.

Kohlensäurebestimmung:

Rohr I.

$$
\begin{gathered}
\mathrm{Hgu}=65,27 \\
\mathrm{Hgo}=41,71 \quad \text { Kaliber }\left\{\begin{array}{l}
40,95 \mathrm{~cm}=87 \mathrm{~cm} \quad \mathrm{~T}=16,0^{\circ} \mathrm{C} . \\
1 \mathrm{~cm}=2,206 \mathrm{ccm} \quad \mathrm{Ba}=757,5 \mathrm{~mm}
\end{array}\right. \\
\mathrm{V}=88,90 \mathrm{~cm} ; \mathrm{P}=506,94 \mathrm{~mm} . \\
\text { Reducirtes Gesammtgas }=56,019 \mathrm{ccm} .
\end{gathered}
$$

Nach Absorption der Kohlensäure:

$$
\begin{aligned}
& \begin{aligned}
\mathrm{Hgu} & =65,63 \\
\mathrm{Hgo} & =18,71 \\
\mathrm{KOH} & =17,44
\end{aligned} \quad \text { Kaliber }\left\{\begin{array}{l}
16,99 \mathrm{~cm}=36 \mathrm{~cm} \\
1 \mathrm{~cm}=2,112 \mathrm{ccm} \quad \mathrm{Ba}=760,0 \mathrm{~mm} .
\end{array}\right. \\
& \mathrm{V}=36,95 \mathrm{ccm} ; \quad \mathrm{P}=283,53 \text {. } \\
& \text { Reducirtes restirendes Gas }=13,077 \mathrm{ccm} \text {. } \\
& \text { Folglich } 42,942 \mathrm{ccm} \mathrm{CO}_{2}=84,45 \mathrm{mgr} \mathrm{CO}_{2}=115,15 \mathrm{mgr} \stackrel{+}{\mathrm{U}} \\
& =0,0537 \mathrm{gr} \text { Stickstoff. }
\end{aligned}
$$


Rohr II.

$\mathrm{Hgu}=66,25$
$\mathrm{Hgo}=41,76$$\quad$ Kaliber $\left\{\begin{array}{l}41,89 \mathrm{~cm}=90 \mathrm{~cm} \quad \mathrm{~T}=16,0^{\circ} \mathrm{C} . \\ 1 \mathrm{~cm}=2,158 \mathrm{~cm} \mathrm{Ba}=758,0 \mathrm{~mm} .\end{array}\right.$

V $89,936 \mathrm{ccm} ; \quad \mathrm{P}=498,15 \mathrm{~mm}$.

Reducirtes Gesammtgas $=55,689 \mathrm{~cm}$.

Nach Absorption der Kohlensäure:

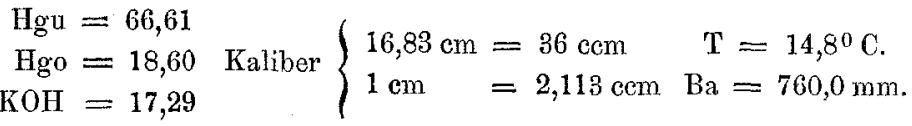

$$
\begin{aligned}
& \mathrm{V}=36,97 \mathrm{~cm} ; \mathrm{P}=272,61 \mathrm{~mm} \text {. } \\
& \text { Reducirtes restirendes Gas }=12,58 \mathrm{ccm} \text {. }
\end{aligned}
$$

Folglich: $43,11 \mathrm{ccm} \mathrm{CO} 0_{2}=84,78 \mathrm{mgr} \mathrm{CO}_{2}=115,61 \mathrm{mgr} \stackrel{+}{\mathrm{U}}$

$$
=0,05395 \mathrm{gr} \text { Stickstoff. }
$$

Mittel: $0,05382 \mathrm{gr}$ Stickstoff.

$5 \mathrm{ccm}=0,0588 \mathrm{gr}$ Stickstoff.

Harn enthält 1,176\% Stickstoff in Harnstoff.

\section{Versuchsserie H.}

\section{Harn III.}

Harnstoffbestimmung nach Bunsen nach Entfernung der durch

Phosphorwolframsäure fällbaren Extractivstoffe. $200,2 \mathrm{ccm}$ Harn

$$
\begin{array}{ll}
20,0 \quad \text { Salzsäure } \\
90,0 \text { " Phosphorwolframsäure }
\end{array}
$$

$310,2 \mathrm{ccm}$ Mischung.

$200,2 \mathrm{ccm}$ des Filtrates $=129,2 \mathrm{ccm}$ Harn wurden mit Kalkpulver $\left(\mathrm{Ca}(\mathrm{OH})_{2}\right)$ zerrieben und alkalisch gemacht. Das Filtrat wurde mit gleichem Volum alkalischer $\mathrm{BaCl}_{2}$-lösung versetzt und je $15 \mathrm{ccm}$ eingeschmolzen. $15 \mathrm{ccm}=$ 4,8405 ccm Harn.

Kohlensäurebestimmung:

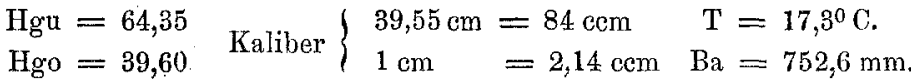

$$
\begin{aligned}
& \mathrm{V}=84,32 \mathrm{ccm} ; \mathrm{P}=489,11 \mathrm{~mm} \text {. } \\
& \text { Reducirtes Gesammtgas }=51,035 \mathrm{ccm} \text {. }
\end{aligned}
$$

Nach Absorption der Kohlensäure:

$$
\begin{aligned}
& \begin{array}{l}
\mathrm{Hgu}=64,65 \\
\mathrm{Hgo}=21,70 \\
\mathrm{KOH}=20,45
\end{array} \text { Kaliber }\left\{\begin{array}{l}
19,85 \mathrm{~cm}=42 \mathrm{ccm} \quad \mathrm{T}=16,8^{\circ} \mathrm{C} . \\
1 \mathrm{~cm}=2,11 \mathrm{~cm} \mathrm{Ba}=752,2 \mathrm{~mm} .
\end{array}\right. \\
& \mathrm{V}=43,266 \mathrm{~cm} ; \mathrm{P}=314,54 \mathrm{~mm} \text {. } \\
& \text { Reducirtes restirendes Gas }=16,87 \mathrm{~cm} \text {, }
\end{aligned}
$$


Folglich: $34,165 \mathrm{ccm} \mathrm{CO}=67,19 \mathrm{mgr} \mathrm{CO}_{2}=0,04276 \mathrm{mgr}$ Stickstoff.

Der Harn enthält also $0,883 \%$ Stickstoff in Harnstoff.

\section{Versuchsserie J.}

\section{Harn IV.}

Harnstoffbestimmung nach Bunsen nach Entfernung der durch Phosphorwolframsäure fällbaren Extractivstoffe.

$$
\begin{array}{rll}
200,2 \mathrm{ccm} & \text { Harn } \\
20,0 & \Rightarrow & \text { Salzsäure } \\
120,0 & \Rightarrow & \text { Phosphorwolframsäure }
\end{array}
$$

$340,2 \mathrm{ccm}$ Mischung.

$200,2 \mathrm{ccm}$ des Filtrates $=117,8 \mathrm{ccm}$ Harn wurden mit Kalkpulver $\left(\mathrm{Ca}(\mathrm{OH})_{2}\right)$ zerrieben und alkalisch gemacht. Das. Filtrat wurde mit gleichem Volum alkalischer $\mathrm{Cl}_{2} \mathrm{Ba}$-lösung versetzt und je $15 \mathrm{~cm}$ eingeschmolzen. $15 \mathrm{ccm}$ $=4,4136 \mathrm{ccm}$ Harn.

Kohlensäurebestimmung:

$$
\begin{gathered}
\text { Hgu }=65,15 \quad \text { Kaliber }\left\{\begin{array}{l}
41,89 \mathrm{~cm}=90 \mathrm{~cm} \quad \mathrm{~T}=17,3^{0} \mathrm{C} . \\
1 \mathrm{~cm}=2,21 \quad \mathrm{Ba}=752,6 \mathrm{~mm} ; \\
\text { Hgo }=41,95 \quad \mathrm{~V}=90,354 \text { ecm; } \mathrm{P}=504,36 \mathrm{~mm} .
\end{array}\right. \\
\text { Reducirtes Gesammtgas }=56,392 \mathrm{ccm} .
\end{gathered}
$$

Nach Absorption der Kohlensäure:

$$
\begin{gathered}
\mathrm{Hgu}=65,45 \\
\mathrm{Hgo}=22,85 \\
\mathrm{KOH}=21,56
\end{gathered} \quad \text { Kaliber }\left\{\begin{array}{l}
21,05 \mathrm{~cm}=45 \mathrm{ccm} \quad \mathrm{T}=16,8^{0} \mathrm{c} . \\
1 \mathrm{~cm}=2,14 \mathrm{~cm} \quad \mathrm{Ba}=752,2 \mathrm{~mm} ; \\
\mathrm{V}=46,09 \mathrm{ccm} ; \mathrm{P}=318,00 \mathrm{~mm} .
\end{array}\right.
$$

Folglich : $38,224 \mathrm{~cm} \mathrm{CO}_{2}=75,17 \mathrm{mgr} \mathrm{CO}_{2}=0,04784 \mathrm{gr}$ Stickstoff.

Der Harn enthält also $1,084 \%$ Stickstoff in Harnstoff.

\section{Berichtigungen qn unseren Anfsätzen in Band XXXVIIl :}

p. 573 Zeile 16 von unten lies "misst" statt "meist".

p. 622 Zeile 4 von oben lies "noch weiter" statt "noch weit".

Pfl. u. B. 